\title{
Electron-hole asymmetry and two-state lasing in quantum dot lasers
}

\author{
E. A. Viktorov and Paul Mandel \\ Optique Nonlinéaire Thréorique, Université Libre de Bruxelles, Campus Plaine Code Postale 231, B-1050 \\ Bruxelles, Belgium \\ Yann Tanguy, John Houlihan, and Guillaume Huyet \\ Optoelectronics Research Group, Tyndall National Institute, National University of Ireland, Cork, Ireland
}

(Received 8 March 2005; accepted 2 June 2005; published online 29 July 2005)

\begin{abstract}
We study the decrease of the ground-state output with increasing current in two-state quantum dot lasing. We show that the asymmetry in the thermal population redistribution breaks the symmetric dynamical evolution of the electron-hole pairs. This fully explains the transition from two-state to single-state lasing observed experimentally. The model also reproduces the temperature dependence of the two-state lasing. (C) 2005 American Institute of Physics. [DOI: 10.1063/1.1995947]
\end{abstract}

Laser devices based on self-assembled quantum dots are a promising source of new physics. ${ }^{1}$ The three-dimensional (3D) confinement of the carriers induces a discrete density of states that implies, for instance, a reduction of the threshold current, ${ }^{2}$ a low chirp, a weak temperature dependence, ${ }^{3}$ and a reduced sensitivity to optical feedback ${ }^{4}$ at telecom wavelengths on GaAs substrates. The recombination of groundstate (GS) electrons and holes leads to GS lasing but recombination of excited-states (ES) electrons and holes can also lead to lasing at lower wavelengths. The occurrence of a secondary threshold, involving a second electron-hole pair of levels, was predicted for a sufficiently high bias. ${ }^{5}$ Increasing the current further, the ES will eventually become the only surviving line at the expense of the GS transition. ${ }^{6-9}$ For injection rates exceeding the second threshold, theory only predicts up to now that the GS intensity becomes a constant while experiments display a reduction down to zero of the GS emission. The aim of this Letter is to analyze both experimentally and theoretically this behavior.

The self-organized quantum dot (QD) active region heterostructure consisted of six InGaAs QD layers embedded in quantum well using dots in a well (DWELL) technology. ${ }^{10}$ Single transverse mode ridge waveguide lasers were fabricated with lengths of $750 \mu \mathrm{m}, 1 \mathrm{~mm}, 1.5 \mathrm{~mm}$, and $2 \mathrm{~mm}$, ridge widths ranging from 3 to $5 \mu \mathrm{m}$, and a depth of $0.9 \mu \mathrm{m}$. Both facets were left uncoated. These devices were mounted epitaxial side up on Peltier-controlled copper heat sinks. While long devices emitted in the GS near $1310 \mathrm{~nm}$, 1-mm-long devices emitted first in the GS but then also in the ES $(1240 \mathrm{~nm})$. The shortest devices emitted in the first excited state for all currents. The light-current curves, characteristic of a 1-mm-device obtained in both $\mathrm{cw}$ and pulsed regimes, are shown in Fig. 1. In both cases, as the injection current increases, there is a current range where the power in GS decreased while the power state in ES increased as previously observed. $^{6-9}$ It is also worth to note that the differential efficiency is larger for the ES, as shown on Fig. 1, highlighting its increased density of states. Operation in the pulsed regime is necessary to remove the role of thermal effects. Figure 1 corresponds to a pulse width of $100 \mathrm{~ns}$ duration with a repetition rate of $100 \mathrm{kHz}$. We verified that the behavior is similar with pulse widths down to $6 \mathrm{~ns}$. The qualitative invariance of the dynamical response with respect to the pulse duration indicates that Joule heating effects do not play a role and cannot explain the decreasing power in GS. Both the GS and ES lasing thresholds were measured with pulse widths of $100 \mathrm{~ns}$ as a function of the device temperature. Above $60{ }^{\circ} \mathrm{C}$ the laser operated only in the excited state, while both states lased below this temperature. For temperatures ranging from 30 to $60{ }^{\circ} \mathrm{C}$, the GS (ES) threshold increased (decreased) almost linearly with a slope of $2.8 \mathrm{~mA}$ /degree (5.3 $\mathrm{mA} /$ degree) .

To explain these experimental results, we consider a rate equation model for the electron and hole populations in both the ground and excited states (see Fig. 2). The indices $g$ and $e$ refer to the ground and the excited levels of the electrons and holes. The two lasing transitions, $E^{g}=0.945 \mathrm{eV}$ and $E^{e}$ $=1.0 \mathrm{eV}$, differ by $\Delta=55 \mathrm{meV}$. Assuming that the electron mass is ten times the hole mass, we estimate the hole and electron energy level spacing as $\Delta E_{h} \simeq 5 \mathrm{meV}$ and $\Delta E_{e}$ $\simeq 50 \mathrm{meV}$. The equations for the electron and hole probabilities in GS, $n_{e, h}^{g}$, and ES, $n_{e, h}^{e}$, and for the intensities $I_{g, e}$ are

$$
\begin{aligned}
& \dot{I}_{g}=\left[2 g_{0}^{g}\left(n_{e}^{g}+n_{h}^{g}-1\right)-1\right] I_{g}, \\
& \dot{I}_{e}=\left[4 g_{0}^{e}\left(n_{e}^{e}+n_{h}^{e}-1\right)-1\right] I_{e}, \\
& \dot{n}_{e, h}^{g}=\eta\left[2 J_{e, h}^{g}-\gamma_{e, h}^{g} n_{e, h}^{g}-\left(n_{e}^{g}+n_{h}^{g}-1\right) I_{g}\right], \\
& \dot{n}_{e, h}^{e}=\eta\left[J_{e, h}^{e}-\gamma_{e, h}^{e} n_{e, h}^{e}-\left(n_{e}^{e}+n_{h}^{e}-1\right) I_{e}\right], \\
& J_{e, h}^{g}=B_{e, h} n_{e, h}^{e}\left(1-n_{e, h}^{g}\right)-C_{e, h} n_{e, h}^{g}\left(1-n_{e, h}^{e}\right),
\end{aligned}
$$

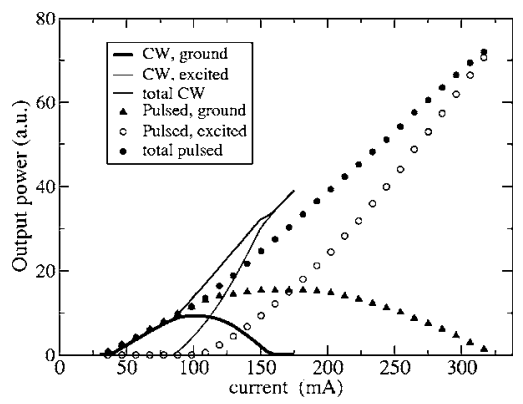

FIG. 1. Characteristic $L-I$ curves for the quantum dot laser in pulsed and $\mathrm{cw}$ regimes. 


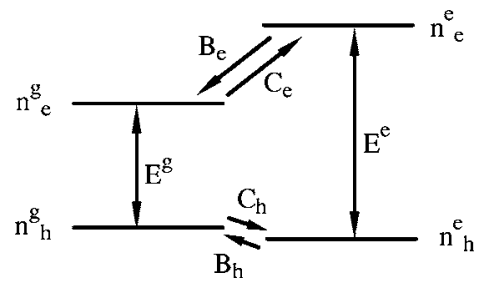

FIG. 2. Energy level scheme.

$$
J_{e, h}^{e}=J_{e, h}\left(1-n_{e, h}^{e}\right)-J_{e, h}^{g},
$$

where $g_{0}^{g, e}$ are the effective gain factors scaled to the cavity losses. For simplicity, we assume these gain factors to be identical for both GS and ES. The dimensionless time is $\tau$ $=t / \tau_{p}$ where $\tau_{p}=2 L / v$ is the photon lifetime. The nonradiative carrier lifetime $\tau_{n}$ is included in the dimensionless parameter $\eta=\tau_{p} / \tau_{n}=2 \times 10^{-3}$. The coefficients 2 and 4 in Eqs. (1) and (2) account for the twofold and fourfold degeneracy of the ground and excited states, respectively. The sources $J_{e, h}^{g, e}$ represent the pumping mechanisms and the capture/ escape processes. The terms $1-n_{e, h}^{g, e}$ describe Pauli blocking. We assume a direct pumping of the excited state from the wetting layer represented by the rates $J_{e, h}$ in Eq. (6) and a nonradiative decay ${ }^{11}$ from the ES to the GS represented by the rates $B_{e, h}$ in the Eq. (5). We also assume that $J_{e, h}=J$ and $B_{e, h}=B$. The underlying physical processes contributing to $B_{e, h}$ have commonly been associated with either carrier-carrier ${ }^{12}$ or carrier-phonon scattering. ${ }^{13-17}$ Assuming a lifetime of $1 \mathrm{~ns}$ for the carriers and $\tau_{c}=7 \mathrm{ps}$, we obtain $B_{e, h}=\tau_{n} / \tau_{c} \approx 150$.

To determine the escape coefficients $C_{e, h}$, we notice that in the absence of pumping and interaction with the field, populations eventually reach equilibrium. Assuming a spontaneous emission rate much smaller than the capture and thermal escape processes (i.e., $B_{e, h}, C_{e, h} \gg 1$ ), a stationary solution for the carrier dynamical evolution is obtained by enforcing the detailed balance condition $J_{e, h}^{g, e}=0 .{ }^{18}$ Inserting a quasiequilibrium Fermi distribution leads to the Kramer relation ${ }^{19}$ linking the capture $B_{e, h}$ and the escape $C_{e, h}$ rates

$$
C_{e, h}=B_{e, h} \exp \left(-\Delta E_{e, h} / k_{B} T\right),
$$

where $\Delta E_{e, h}$ represents the positive energy separation between the excited and the ground state levels, $k_{B}$ is the Boltzmann constant, and $T$ the plasma temperature.

Let us stress that the thermal redistribution occurs on different time scales for holes and electrons. Using Eq. (7) with $\Delta E_{h}=5 \mathrm{meV}$ and $\Delta E_{e}=50 \mathrm{meV}$ at room temperature $\left(k_{B} T=25 \mathrm{meV}\right)$ we have $C_{e} \approx 0.1 B$ and $C_{h} \approx 0.8 B$. The difference between $C_{e}$ and $C_{h}$ is significant and indicates that thermal processes break the symmetric evolution of electronhole pairs. This results in a dramatic difference between the redistribution rates of the two types of carriers. While the electron populations are strongly dissymmetric due to the relatively small value of $C_{e}$, the two hole populations are almost equal. Consequently, the hole reservoir acts as one effective level in the limit where $\Delta E_{h} \ll k_{B} T$ and $\Delta E_{e} \gg k_{B} T$, and the approximations $C_{e}=0$ and $C_{h}=B$ can be used. The equation determining the hole population redistribution expresses a balance between pumping and population transfer between the two hole levels: $J_{h}^{e}=J\left(1-n_{h}^{e}\right)-B\left(n_{h}^{e}-n_{h}^{g}\right)$ and $J_{h}^{g}=B\left(n_{h}^{e}-n_{h}^{g}\right)$. With this approximation, and rewriting Eqs. (3) and (4) in terms of the sum and the difference of the hole populations, one notices that the difference $n_{-}=n_{h}^{e}-n_{h}^{g}$ has a decay time of $1 /(3 \eta B) \equiv \varepsilon / \eta$ while the sum has a decay time of $1 / \eta$. Since $B \gg 1$, the population difference can be adiabatically eliminated and one finds that $n_{-}=0$ to first order in $\epsilon$.

Recent papers have focused on the two-state lasing dynamic $^{7-9}$ using either rate equations or a master equation model. ${ }^{20}$ In these models, the nonradiative decay from the ES to the GS rules the dynamical evolution of the field and, in particular, determines the possibility of two-state lasing. However, the thermal population of the excited levels is neglected in the master equation model ${ }^{20}$ because it was derived for very low temperatures. Thus, while $C_{e}$ vanishes in first approximation, $C_{h}$ remains finite at room temperature.

Our experimental observations can be explained by taking into account the two main features of the redistribution rates calculated above: the thermal balance between the two hole levels $\left(C_{h} \simeq B_{h}\right)$ and the electron-hole asymmetry $\left(C_{e}\right.$ $\left.\ll C_{h}\right)$.

When lasing occurs on both GS and ES transitions, the thermal balance between the two hole levels implies that both fields compete for the same holes. Since the effective gain of the ES transition is twice the effective gain of the GS transition, the ES field increases with pump at the expense of the GS field.

The electron-hole asymmetry for the redistribution rates has an important consequence. Equations (1)-(6) describe a limited set of levels: For the electrons and holes, only the ground state and the first of the excited state levels are retained. All the other excited states have been neglected. As a result, Eqs. (1)-(6) preserve charge neutrality for the ground state only if $B_{e}=B_{h}$ and $C_{e}=C_{h}$. In this case, the description of the system can be based on the excitonic energy states as recently proposed. ${ }^{7-9}$ However, if $C_{e} \ll C_{h}$, the electron-hole symmetry is broken in the dynamical equations: Equations (1)-(6) lead to a value for $n_{e}^{g}$ that is too large to preserve charge neutrality in the case of GS emission. Therefore, we must consider how negatively charged excitons contribute to the two-state lasing. The fact that describing QD lasers using only average carrier population may be insufficient has already been pointed out. ${ }^{21}$

There are two recombination mechanisms. One possibility is that the negatively charged exciton captures an additional hole and forms a biexciton with a subsequent recombination. However, it has been shown ${ }^{7-9}$ that this excitonic picture leads to a saturation of the GS lasing with increasing pump instead of the observed decrease. The other possibility is that in the negatively charged exciton one electron-hole pair recombines. After annihilation, there remains one photon and one electron. The excess electron escapes the level via a re-emission process. This depletes the carrier population for the ground state and the GS intensity decreases. Consequently, a linear decay rate of the carrier populations $\gamma_{e, h}^{g, e} n_{e, h}^{g, e}$ is necessary to reproduce the experimentally observed decrease of the GS emission. The phenomenological rates $\gamma_{e, h}^{g, e}$ in Eqs. (1)-(6) account for a number of possible processes like thermal reemission in the wetting layer, Auger depopulation effect, or relatively long electron spin relaxations recently considered in Ref. 22. To simplify the analysis, we use $\gamma_{e, h}^{g, e}=1$. We have also verified that the inclusion of a radiative bilinear decay term involving the product of the electron and hole populations does not lead to qualitative changes of the results.

to AIP license or copyright, see http://apl.aip.org/apl/copyright.jsp 


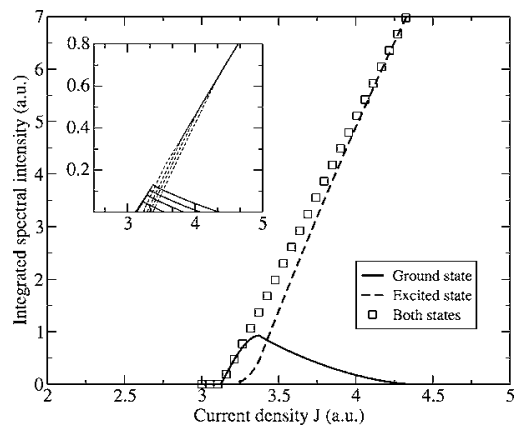

FIG. 3. Spectrally averaged $L-I$ curves for the ground and excited states. Inset: a sample of single mode curves.

In order to reproduce the multimode spectra observed experimentally, we consider 20 pairs of modes for the ground and the excited states, neglecting both spectral and spatial interaction between modes belonging to the same transition. We estimate the broadening of the transition to be $\Delta E$ $\approx 60 \mathrm{meV}$ and model it by a parabolic gain curve around the maximum value $g_{0}^{g, e}=0.785$. In these equations, we neglect both spectral and spatial interactions between modes, which is justified for single transverse mode devices given the strong inhomogeneous broadening that usually occurs in self-organized quantum dot materials. As in the experiment, the emission occurs from the ground state at threshold and increases until threshold occurs for the excited state (Fig. 3). Above this point, rollover occurs for the excited state until it switches off completely. Emission now occurs at excited state wavelengths only. We also verified that, for a temperature ranging from 22 to $30 \mathrm{meV}$, the GS (ES) threshold increased (decreased) linearly.

In summary, we have presented a modified rate equation model taking into account explicitly population thermal redistribution and explained the decay of the GS with the increasing current. The temperature dependence of the two lasing thresholds is also well reproduced by the model.
The authors acknowledge partial support of the Fonds National de la Recherche Scientifique (Belgium), the Interuniversity Attraction Pole Programme-Belgian Science Policy, the Science Foundation Ireland (SFI) under Grant No. SFI/01/F.1/CO13, and the Irish Higher Education Authorities under the Program for Research in Third Level Institutions. The wafers were supplied by Zia Laser Inc. and processed by Brian Corbett at NMRC, Cork.

${ }^{1}$ D. Bimberg, M. Grundmann, and N. N. Ledentsov, Quantum Dot Heterostructures (Wiley, New York, 1999).

${ }^{2}$ R. S. C. Ribbat, M. Grundmann, N. N. Ledentsov, and D. Bimberg, Appl. Phys. Lett. 78, 1104 (2001).

${ }^{3}$ O. B. Shchekin and D. G. Deppe, IEEE Photonics Technol. Lett. 14, 1231 (2002).

${ }^{4}$ D. O'Brien, S. P. Hegarty, G. Huyet, J. G. McInerney, T. Kettler, M. Laemmlin, D. Bimberg, V. M. Ustinov, A. E. Zhukov, S. S. Mikhrin, and A. R. Kovsh, Electron. Lett. 39, 1819 (2003).

${ }^{5}$ L. V. Asryan, M. Grundmann, N. N. Ledentsov, O. Stier, R. A. Suris, and D. Bimberg, IEEE J. Quantum Electron. 37, 418 (2001).

${ }^{6}$ G. Park, D. L. Huffaker, Z. Zou, O. B. Shchekin, and D. G. Deppe, IEEE Photonics Technol. Lett. 11, 301 (1999).

${ }^{7}$ A. Markus, J. X. Chen, C. Paranthoen, A. Fiore, C. Platz, and O. GauthierLafaye, Appl. Phys. Lett. 82, 1818 (2003).

${ }^{8}$ A. Markus, J. X. Chen, O. Gauthier-Lafaye, J.-G. Provost, C. Paranthoën, and A. Fiore, IEEE J. Sel. Top. Quantum Electron. 9, 1308 (2003).

${ }^{9}$ A. Markus and A. Fiore, Phys. Status Solidi A 201, 338 (2004).

${ }^{10}$ T. Newell, D. Bosser, A. Stintz, B. Fuchs, K. Malloy, and L. Lester, IEEE Photonics Technol. Lett. 11, 1527 (1999).

${ }^{11}$ K. Mukai, N. Ohtsuka, and M. Sugawara, Jpn. J. Appl. Phys., Part 2 35, L262 (1996).

${ }^{12}$ U. Bockelmann and T. Egeler, Phys. Rev. B 46, 15574 (1992).

${ }^{13}$ U. Bockelmann and G. Bastard, Phys. Rev. B 42, 8947 (1990).

${ }^{14}$ H. Benisty, C. M. Sotomayor-Torršs, and C. Weisbuch, Phys. Rev. B 44, 10945 (1991).

${ }^{15}$ T. Inoshita and H. Sakaki, Physica B 227, 373 (1996).

${ }^{16}$ T. Inoshita and H. Sakaki, Phys. Rev. B 56, R4355 (1997).

${ }^{17}$ S. Hameau, Y. Guldner, O. Verzelen, R. Ferreira, G. Bastard, J. Zeman, A. Lemaître, and J. M. Gérard, Phys. Rev. Lett. 83, 4152 (1999).

${ }^{18}$ H. Jiang and J. Singh, J. Appl. Phys. 85, 7438 (1999).

${ }^{19}$ L. V. Asryan and R. A. Suris, Semicond. Sci. Technol. 11, 554 (1996).

${ }^{20}$ M. Grundmann and D. Bimberg, Phys. Rev. B 55, 9740 (1997).

${ }^{21}$ M. Grundmann, R. Heitz, and D. Bimberg, Phys. Solid State 40, 772 (1998).

${ }^{22}$ C. Cao and D. G. Deppe, Appl. Phys. Lett. 84, 2736 (2004). 